\title{
GaSb-based Solar Cells for Multi-junction Integration on Si substrates
}

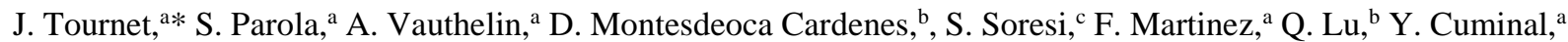
P.J. Carrington, ${ }^{\mathrm{d}}{ }^{\mathrm{J}}$. Décobert, ${ }^{\mathrm{c}}$ A. Krier, ${ }^{\mathrm{b}}$ Y. Rouillard ${ }^{\mathrm{a}}$ and E. Tourniéa*

${ }^{a}$ IES, Univ. Montpellier, CNRS, F- 34000, Montpellier, France

b Physics Department, Lancaster University, Lancaster LA1 4YB, United Kingdom

c III-V Lab, 1 Avenue Augustin Fresnel, 97167, Palaiseau, France

d Department of Engineering, Lancaster University, Lancaster LA1 4YW, United Kingdom

* corresponding authors: julie.tournet@umontpellier.fr, eric.tournie@umontpellier.fr

\begin{abstract}
:
We report on the first single-junction GaSb solar cell epitaxially grown on a Si substrate. A control stand-alone GaSb solar cell was primarily fabricated, which demonstrated a 5.90\% efficiency (AM1.5G). The preparation, growth and manufacturing procedures were then adapted to create the GaSb-on-Si solar cell. The hybrid device resulted in a degraded efficiency for which comparison between experimental and simulated data revealed dominant non-radiative recombination processes. Material and electrical characterization also highlighted the impact of anti-phase domains and boundaries and threading dislocation density on the shunt resistance of the cell. Nevertheless, the GaSb-on-Si cell performance is close to recent results on the integration of GaSb solar cells on GaAs, despite a much larger lattice mismatch (12\% vs 8\%). Routes for improvement, concerning the material quality and cell structure, are proposed. This work lays the foundations of a GaSb-based multi-junction solar cell monolithically integrated on $\mathrm{Si}$.
\end{abstract}




\title{
GaSb-based Solar Cells for Multi-junction Integration on Si substrates
}

\author{
J. Tournet, ${ }^{\text {* }}$ S. Parola, ${ }^{\mathrm{a}}$ A. Vauthelin, ${ }^{\mathrm{a}}$ D. Montesdeoca Cardenes,, , S. Soresi, ${ }^{\mathrm{c}}$ F. Martinez, ${ }^{\mathrm{a}}$ Q. Lu, ${ }^{\mathrm{b}}$ Y. Cuminal, ${ }^{\mathrm{a}}$ \\ P.J. Carrington, ${ }^{\mathrm{d}}{ }^{\mathrm{J}}$. Décobert, ${ }^{\mathrm{c}}$ A. Krier, ${ }^{\mathrm{b}}$ Y. Rouillard ${ }^{\mathrm{a}}$ and E. Tourniéa* \\ ${ }^{a}$ IES, Univ. Montpellier, CNRS, F- 34000, Montpellier, France \\ b Physics Department, Lancaster University, Lancaster LA1 4YB, United Kingdom \\ c III-V Lab, 1 Avenue Augustin Fresnel, 97167, Palaiseau, France \\ ${ }^{\mathrm{d}}$ Department of Engineering, Lancaster University, Lancaster LA1 4YW, United Kingdom \\ * corresponding authors: julie.tournet@umontpellier.fr, eric.tournie@umontpellier.fr
}

\section{Introduction}

Historically, silicon is the most used material in semiconductor-based devices and its dominance looks set to continue. In addition to its abundance on Earth's surface, it is a material whose processing techniques are well established in industry. Its high availability and manufacturing maturity make it an affordable technological solution and explain its dominance in the solar energy market. Broadly used for decades, it has proven reliability in photovoltaic systems, especially with regards to lifetime requirements. However, its indirect bandgap presents a major disadvantage, since a significant part of the photo-generated energy is lost through phonon emission. This is reflected in the performances reported for crystalline Si solar cells: efficiencies have plateaued around $25 \%$ with the most recent development being a 26.7\% efficiency solar cell (AM1.5G) fabricated by Kaneka corporation [1]. Kaneka's report represents a $1.7 \%$ absolute improvement over the efficiencies obtained almost 20 years ago [2]. Even under concentrated light, there has been no advancement since 2005 with a record efficiency still set at $27.6 \%$ under 92 suns [3]. Despite the majority of the existing commercial solar cells being made of Si, it is understandably not the material of choice in the pursuit of the highest possible solar efficiency. Unlike Si, most III-V materials exhibit a direct bandgap. The development of technologies such as concentrated photovoltaics has allowed for a reduction in size required for a solar cell, opening new opportunities for these more expensive, yet more efficient, materials. The superior radiation resistance and thermal stability of III-V materials makes them particularly favorable candidates for solar applications in space. However, despite steady increases in their reported record efficiencies [2], III-V solar cells have faced economic difficulties in recent years [4]. The power gain III-V cells generate does not yet balance the added fabrication costs. Although the use of solar concentrators has enabled cost reductions of III-V systems down to $0.59 \$ / \mathrm{W}_{\mathrm{p}}$ (whole module), it is still well above the $0.24 \$ / \mathrm{W}_{\mathrm{p}}$ of a standard Si panel [5]. The option of combining the advantages of both materials is an interesting one, allowing cost reduction and facilitating higher production volumes. The integration of III-V cells on Si substrates is thus eagerly awaited. Several techniques, associated with different challenges, have been developed in the past decades. Here, we focus on the monolithic integration of III-V on Si. Firstly, we discuss the advantages of III-Sb structures for heteroepitaxy on $\mathrm{Si}$, describing our research strategy for integration. Secondly, we detail the growth sequences and processing steps that were followed to produce a control GaSb-on-GaSb solar cell and a hybrid GaSb-on-Si solar cell. We further discuss the performance of both devices and conclude on the potential of III-Sb for high efficiency III-V-on-Si solar cells.

\section{Monolithic integration of III-Sb on Si}

The hetero-epitaxy of III-V compounds on Si faces three main challenges. First, the significant latticemismatch generates numerous dislocations which are detrimental to the performance of the electronic device. If the threading dislocations propagate into the active region of the solar cell, they can act as non-radiative recombination centers, reducing the minority carrier lifetime. Second, the growth of a polar material on a nonpolar material induces the creation of anti-phase domains at the boundaries of which non-radiative recombination can also occur. The emergence of anti-phase domains can be prevented by the use of miscut substrates which creates a double-stepped starting surface, but is difficult to achieve in realistic lab conditions. Finally, the discrepancy in thermal expansion coefficient can lead to cracks and film bending during and after growth. Multiple growth techniques have been developed in order to produce strain free III-V layers grown on Si with low threading dislocation density (TDD). In practice, Yamaguchi et al have shown that a TDD below $3 \cdot 10^{5} \mathrm{~cm}^{-2}$ was sufficient to drastically limit non-radiative recombination losses in metamorphic solar cells [6]. At this dislocation density, the spacing between dislocations becomes larger than the diffusion length, significantly reducing the recombination losses. The thick buffer and compositionally graded buffer techniques aim at the controlled 
formation and glide of the dislocations in order to achieve low TDDs. These techniques encourage the propagation of dislocations, through the thickness or by inducing increasing strain, until they annihilate by encountering others. An alternative technique is the introduction of strained superlattices (SLS), which encourages the bending and coalescence of dislocations at the hetero-interfaces. Such structures are also helpful in eliminating segregating contaminants. Finally, thermal cycle growth (TCG) and thermal cycle annealing (TCA) can be used to further encourage the dislocations' glide and annihilation. At elevated temperatures, the grown films undergo multiple compression and tension forces, causing the dislocations to move in multiple directions, increasing their chances of encountering other dislocations and annihilating. This method, however, has a major drawback in the case of III-V-on-Si epitaxy: the difference in thermal expansion could generate more dislocations and cracks in the material. Despite all these techniques, the $10^{5} \mathrm{~cm}^{-2}$ TDD range is hardly achievable in practice. Recently, Yaung et al achieved a $5.25 \cdot 10^{6} \mathrm{~cm}^{-2}$ TDD with a $40 \mathrm{~nm} \mathrm{GaP}$ nucleation layer, followed by a $550 \mathrm{~nm}$ GaP buffer and a $3.6 \mu \mathrm{m}$ graded $\mathrm{GaAs}_{\mathrm{y}} \mathrm{P}_{1-\mathrm{y}}$ buffer made of 28 steps [7]. The structure was grown by Molecular Beam Epitaxy (MBE) on an on-axis Si substrate. The resulting $\mathrm{GaAs}_{0.76} \mathrm{P}_{0.24}$ single-junction cell demonstrated a $15.3 \%$ efficiency [8]. This result is still below the record efficiency GaAs single-junction cells on Si ([9], [10], [11]) but demonstrates the advantage of using a simplified growth procedure to achieve a similar TDD.

Unlike Arsenides ([9], [10], [11], [12], [13], [14]) and Phosphides ([7], [8]), Antimonides have received only limited attention in the context of photovoltaic research. This is particularly true in the case of III-V-on-Si solar cells, yet III-Sbs have already demonstrated their potential for other integrated opto-electronic devices [15], [16], [17], [18]. Such materials take advantage of the creation of a $90^{\circ}$ misfit dislocations array when using an AlSb initiation layer on the Si [19]. This interfacial misfit array efficiently relieves the strain at the interface. Akahane et al also highlighted the contribution of the AlSb nucleation layer in terms of surface morphology [20]. Rodriguez et al further showed that the optimal nucleation conditions were $4 \mathrm{ML}$ of $\mathrm{AlSb}$ grown at $450^{\circ} \mathrm{C}$ [21]. Post-growth annealing proved to further enhance the material quality, lowering the FWHM value down to 235 arcsec for a 1 $\mu \mathrm{m}$ GaSb-on-AlSb-on-Si layer, close to values obtained for similar thicknesses of Ge-on-Si [22] and GaAs-on-Si [23]. This is in spite of a significantly larger lattice-mismatch between the III-V compound and the $\mathrm{Si}(\sim 12 \%$ vs $\sim 4 \%$ for GaAs). TDDs in the $10^{9} \mathrm{~cm}^{-2}$ range were evaluated for these thin samples [24]. Supported by these observations, we have decided to investigate the growth and processing of GaSb single junction cells on $\mathrm{Si}$. GaSbbased solar cells were recently studied to serve as subcells of multi-junction solar cells [25], [26], [27]. The wide range of alloys lattice-matched to GaSb, combined with the natural broken-gap alignment of the InAs/GaSb tunnel junction [28] could actually allow the fabrication of the entire structure of an all lattice-matched multi-junction cell. The first step of this investigation is the development of state-of-the-art stand-alone GaSb solar cells. We refer to these samples as "control solar cells" in the following sections. The second step is the monolithic integration of such cells onto an inactive Si substrate. We refer to these samples as "hybrid solar cells" below. The development of III-Sb materials for GaSb-based multi-junction cells constitute the third step, which is beyond the scope of this document and treated separately [25].

\section{Experimental details}

The lattice-matched GaSb control solar cells were grown by MBE in a VARIAN GEN II reactor. Upon loading into the growth reactor, 2-inch epi-ready n-type (001) GaSb:Te substrates were deoxidized under Sb flux at $550^{\circ} \mathrm{C}$. The substrate temperature was measured with cross-calibrated thermocouple and pyrometer and confirmed with the $\mathrm{GaSb}(001)-(2 \times 5)$ to $(1 \times 3)$ change of reconstruction [29]. After 20 min of deoxidation, the temperature was lowered to $500^{\circ} \mathrm{C}$ to grow a $200 \mathrm{~nm} \mathrm{GaSb}$ buffer layer. The solar cell growth was then initiated at the same temperature. The GaSb growth rate, set by the Gallium growth rate, was $1 \mathrm{ML} / \mathrm{s}$. The V/III growth rate ratio was kept close to 2. Be and Te were used for $\mathrm{p}$ - and n-type doping respectively. The control cell structure is shown on Fig. 1.(a). It is made of a $500 \mathrm{~nm}$ back surface field (BSF), a $1500 \mathrm{~nm}$ base, a $500 \mathrm{~nm}$ emitter and a $30 \mathrm{~nm}$ window layer. The structure was eventually capped with a p-doped $10 \mathrm{~nm}$ contact layer, which also provided protection to the active region during the subsequent processing steps.

The hybrid solar cells required a specific surface preparation prior to growth. Quarters of 2-inch wafers were first chemically deoxidized [30]. The substrates were quickly introduced into the MBE reactor following a final HF dip. Once under vacuum, the samples were thermally treated by flash annealing at $800^{\circ} \mathrm{C}$ without any impinging flux. The heteroepitaxy was initiated at $450^{\circ} \mathrm{C}$ with $4 \mathrm{ML}$ of AlSb grown at $0.35 \mathrm{ML} / \mathrm{s}$. The nucleation layers were then covered with $50 \mathrm{~nm}$ of GaSb grown at $1 \mathrm{ML} / \mathrm{s}$. At this point, the substrate temperature was raised to $480^{\circ} \mathrm{C}$ for the growth of a $1000 \mathrm{~nm} \mathrm{GaSb}$ buffer. The solar cell structure was eventually grown as shown on Fig. 1.(b). A $500 \mathrm{~nm} \mathrm{InAs} 0.91 \mathrm{Sb}_{0.09}$ contact layer was grown at $430^{\circ} \mathrm{C}$ to serve as the bottom contact and capped with $50 \mathrm{~nm}$ of GaSb. This alloy composition, lattice-matched to GaSb, will simply be referred to as InAsSb below. 
The temperature was then raised back to $480^{\circ} \mathrm{C}$ for the remainder of the growth consisting of a $1000 \mathrm{~nm} \mathrm{BSF}$, a $1500 \mathrm{~nm}$ base, a $500 \mathrm{~nm}$ emitter, a $30 \mathrm{~nm}$ window and a $10 \mathrm{~nm}$ cap/contact layer. Once again, Be and Te were used for $\mathrm{p}$ - and n-type doping respectively. The InAsSb growth rate was $0.31 \mathrm{ML} / \mathrm{s}$. For both types of cells, the surface was monitored during growth with reflection high energy electron diffraction (RHEED). The crystalline quality was subsequently confirmed with high resolution X-ray diffraction (XRD).

(a)

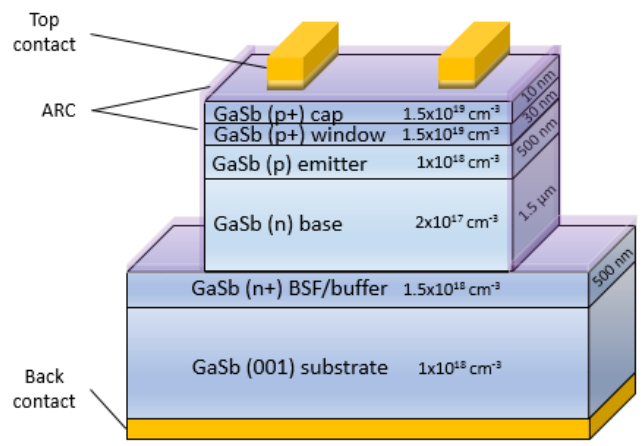

(b)

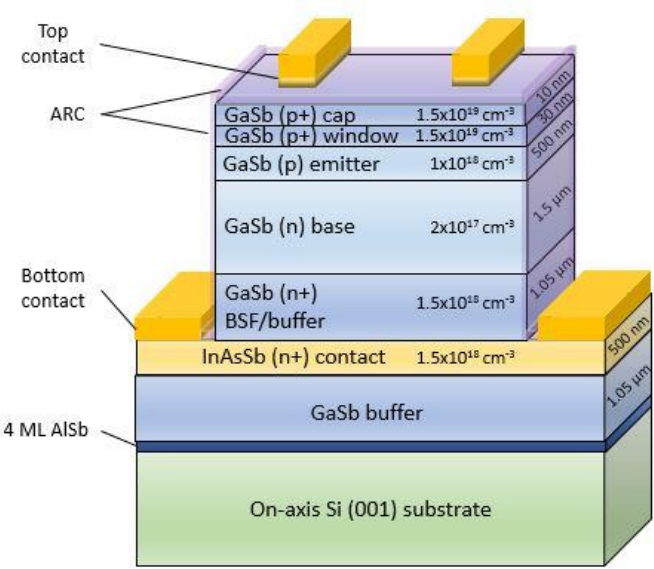

Fig. 1 Schematic structure of the GaSb-on-GaSb control cell (a) and GaSb-on-Si hybrid cell (b).

After growth, $0.5 \mathrm{~cm} \times 0.5 \mathrm{~cm}$ single junction GaSb solar cells were fabricated in a class 10000 clean room at the University of Montpellier. The control solar cell process, developed in collaboration with III-V lab, comprised four steps represented in Fig. 2.(a). Standard clean room techniques, such as UV photolithography, were employed. $\mathrm{Ti} / \mathrm{Au} 30 / 250 \mathrm{~nm}$ were first deposited by electron beam evaporation for the front contacts. $2 \mu \mathrm{m}$ deep trenches were then opened by inductively coupled plasma reactive ion etching (ICP RIE), to isolate the cells from each other. A $214 \mathrm{~nm}$ thick $\mathrm{SiN}_{\mathrm{x}}: \mathrm{H}$ passivation/anti-reflective coating (ARC) layer was later deposited on the top and the sides of the active surface. On some samples, a $291 \mathrm{~nm}$ thick $\mathrm{SiO}_{2}$ layer was used instead. Plasma enhanced chemical vapor deposition (PECVD) and ICP RIE were employed for this step. Finally, Pd/AuGeNi 5/200 nm layers were deposited by sputtering on the whole back surface. For hybrid solar cells, the fabrication procedure had to be adapted to circumvent the high defect density at the Si/III-Sb interface. Keeping the back of the substrate as a bottom contact would indeed induce many electric losses via the hetero-interface. We therefore decided to bring both contacts to the front of the sample, leaving one at the very top of the solar cell and setting the other one at its base, as shown on Fig. 1.(b). The new process flow (shown on Fig. 2.(b)) comprised three steps, namely the mesas etching, the surface and side passivation (214 nm thick $\mathrm{SiN}_{\mathrm{x}}: \mathrm{H}$ ARC) and the metallization (Ti/Au 30/250 $\mathrm{nm}$, top and bottom simultaneously). It was decided here to proceed first to the passivation and then to the contacts deposition in order to avoid any metal from depositing on the sides of the solar cells, leading to electrical short.

At the end of the processing, the samples were cleaved into individual cells and mounted on TO- 8 holders with silver paint. The solar cells bus bars and back/bottom contacts were connected to the TO- 8 pins by microwelding. The solar cells were characterized at the University of Montpellier (France) using a Newport ORIEL Sol 3A solar simulator for the electrical characterization. The cells were mounted on a Peltier element to maintain a temperature of $25^{\circ} \mathrm{C}$ upon illumination. The lamp was calibrated using a reference Si cell and equipped with a filter to provide an AM1.5G illumination. A Keithley 2400 Source Meter was used to measure the electrical parameters of the cells. Control solar cells were also characterized at the University of Lancaster (United Kingdom) with a LOT ORIEL LS0106 solar simulator. The spectral response of the cells was acquired with a set up consisting of a Xenon lamp and an Acton ARC AM-505F spectrometer. The equipment was calibrated with a reference $\mathrm{Si}$ photodiode (BN-DSR-100F, Gigahertz-Optik) and a Ge photodiode (FDG03-CAL, Thorlabs). 
(a) CONTROL SOLAR CELL PROCESS FLOW

(b) HYBRID SOLAR CELL PROCESS FLOW (1) Front metal
deposition

(2) Mesas etching

3 Passivation

(4) Back metal deposition
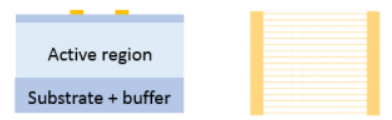

(1) Mesas

etching

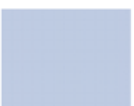

Substrate + buffer
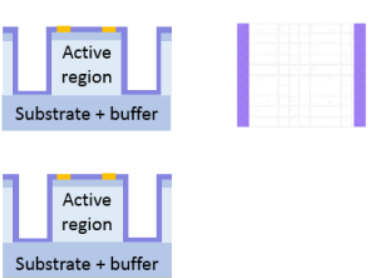

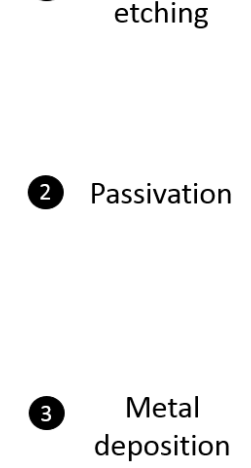

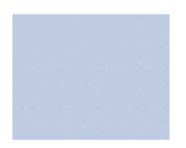
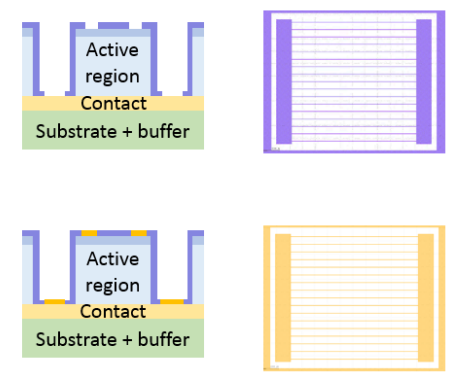

Fig. 2 Schematic illustration of the processing steps for the fabrication of the GaSb-on-GaSb control solar cell (a) and GaSb-on-Si hybrid solar cell (b). For each step, a picture of the corresponding photolithography mask is displayed (right).

\section{GaSb-on-GaSb control solar cell}

In order to realize a state-of-the-art GaSb control cell, we have performed an extensive study of the device, ensuring the stability of the cells over time and the impact of the passivation/ARC. All of the cells mentioned below originated from the same epitaxial sample and were processed at the same time. Three types of cells were fabricated: $\mathrm{SC} 012$ cells with a $\mathrm{SiN}_{\mathrm{x}}: \mathrm{H}$ passivation, $\mathrm{SC} 015$ cells with a $\mathrm{SiO}_{2}$ passivation and $\mathrm{SC} 013$ cells without any passivation. Table I shows a comparison of the results obtained in both measurement sites. The best control solar cell demonstrated a 1 -sun efficiency $(\eta)$ of $5.90 \%$, indicating a reasonably high material quality [31]. The cell had a $280 \mathrm{mV}$ open-circuit voltage $\left(\mathrm{V}_{\mathrm{OC}}\right)$, a $36 \mathrm{~mA} / \mathrm{cm}^{2}$ short-circuit current density $\left(\mathrm{J}_{\mathrm{SC}}\right)$ and a $58.9 \%$ fill factor (FF). The same cell exhibited similar characteristics when measured in Lancaster, four months later, with an efficiency of $5.84 \%$, a $\mathrm{V}_{\mathrm{OC}}$ of $286 \mathrm{mV}$, a $\mathrm{J}_{\mathrm{SC}}$ of $37 \mathrm{~mA} / \mathrm{cm}^{2}$ and a $\mathrm{FF}$ of $55.1 \%$. The shading factor of this particular cell was 5\%. The repeated measurements were consistent for all types of cells, demonstrating the stability of the devices and the reliability of the measurement set-ups. While this is expected for the passivated cells, it is interesting to note that there is not much degradation between the parameters measured in July and November for the unpassivated SC013 cell. This indicates that any oxidation process occurring at the surface of the cell is almost complete from the very beginning and does not worsen significantly over time. Still, we have observed that the passivation layer reduces the dispersion of the JV curves (observed over 8 samples), making the cell fabrication-more reproducible.

TABLE I

CORRELATION BETWEEN THE DISTINCT SITES' MEASUREMENTS

1 -sun efficiency $(\eta)$, fill factor $(\mathrm{FF})$, short-circuit current density $\left(\mathrm{J}_{\mathrm{SC}}\right)$ and open-circuit voltage $\left(\mathrm{V}_{\mathrm{OC}}\right)$ measured for control cells in Montpellier (July) and Lancaster (November).

\begin{tabular}{cccccc}
\hline \hline \multirow{2}{*}{ Cell } & $\begin{array}{c}\text { Measurement } \\
\text { site }\end{array}$ & $\eta(\%)$ & FF & $\begin{array}{c}\mathrm{J}_{\mathrm{SC}} \\
\left(\mathrm{mA} / \mathrm{cm}^{2}\right)\end{array}$ & $\begin{array}{c}\mathrm{V}_{\mathrm{OC}} \\
(\mathrm{mV})\end{array}$ \\
\hline \multirow{2}{*}{ SC012a } & Montpellier & 5.90 & 0.59 & 36 & 280 \\
& Lancaster & 5.84 & 0.55 & 37 & 286 \\
\multirow{2}{*}{ SC015a } & Montpellier & 4.86 & 0.51 & 34 & 280 \\
& Lancaster & 4.75 & 0.48 & 35 & 280 \\
& Montpellier & 4.21 & 0.54 & 28 & 280 \\
& Lancaster & 4.31 & 0.52 & 31 & 270 \\
\hline \hline
\end{tabular}


TABLE II

JV PARAMETERS MEASURED FOR GASB-ON-GASB CONTROL SOLAR CELLS

1 -sun efficiency $(\eta)$, fill factor $(\mathrm{FF})$, short-circuit current density $\left(\mathrm{J}_{\mathrm{SC}}\right)$ and open-circuit voltage $\left(\mathrm{V}_{\mathrm{OC}}\right)$ measured for all types of control cells $(5 \%$ shading factor): with $\mathrm{SiN}_{\mathrm{x}}: \mathrm{H}$ passivation $(\mathrm{SC012})$, with $\mathrm{SiO}_{2}$ passivation ( $\mathrm{SC015}$ ) and without any passivation (SC013). These measurements were made in the University of Lancaster.

\begin{tabular}{ccccc}
\hline \hline Cell type & $\eta(\%)$ & $\mathrm{FF}$ & $\mathrm{J}_{\mathrm{SC}}\left(\mathrm{mA} / \mathrm{cm}^{2}\right)$ & $\mathrm{V}_{\mathrm{OC}}(\mathrm{mV})$ \\
\hline$S C 012 a\left(\mathrm{SiN}_{x}: H\right)$ & 5.84 & 0.55 & 37 & 286 \\
$S C 015 b\left(\mathrm{SiO}_{2}\right)$ & 4.47 & 0.47 & 34 & 280 \\
$S C 013 b(\emptyset)$ & 4.40 & 0.52 & 32 & 270 \\
\hline \hline
\end{tabular}

We will now focus on the results summarized in Table II, corresponding to cells with a shading factor of $5 \%$. It appears that the $\mathrm{SiN}_{\mathrm{x}}: \mathrm{H}$ passivation/ARC yields higher efficiencies. The associated JV curves, shown on Fig. 3 , provide additional data concerning the impact of passivation. The dark JV data were fitted to the two-diode lumped model for an abrupt pn junction [32]. Ideality-of-one dark current $\mathbf{J}_{01}$, ideality-of-two dark current $\mathbf{J}_{02}$ and an estimate of the shunt resistance $\mathrm{R}_{\mathrm{sh}}$ were extracted. The passivated cells exhibited higher shunt resistance $\left(\mathrm{R}_{\mathrm{sh}}\right.$ of $1.7 \times 10^{3} \Omega \mathrm{cm}^{2}$ and $1.3 \times 10^{3} \Omega \mathrm{cm}^{2}$ for SC012a and SC015b respectively, vs $5.3 \times 10^{2} \Omega \mathrm{cm}^{2}$ for SC013b), indicating reduced leakage currents on the surface and edges of the mesas. Particularly in forward bias, the generation-recombination current is lower on the passivated samples $\left(\mathrm{J}_{02}\right.$ of $1.3 \times 10^{-5} \mathrm{~A} / \mathrm{cm}^{2}$ and $1.6 \times 10^{-5} \mathrm{~A} / \mathrm{cm}^{2}$ for SC012a and SC015b respectively) than on the unpassivated one $\left(\mathrm{J}_{02}\right.$ of $3.10 \times 10^{-5} \mathrm{~A} / \mathrm{cm}^{2}$ for SC013b), indicating a longer carrier lifetime. The two types of passivation achieve close results and cannot really be distinguished. The added benefit of the $\mathrm{SiN}_{\mathrm{x}}: \mathrm{H}$ on the 1-sun curve(s) can therefore be attributed to its anti-reflective action. As dark JV curves are similar for SC012a and SC015b in the forward regime, the better performance of the SC012a cell under illumination is due only to the increased generated photocurrent. This is expected from the

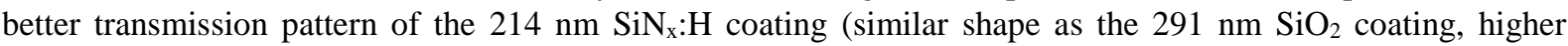
intensity), as simulated from ellipsometry measurements.

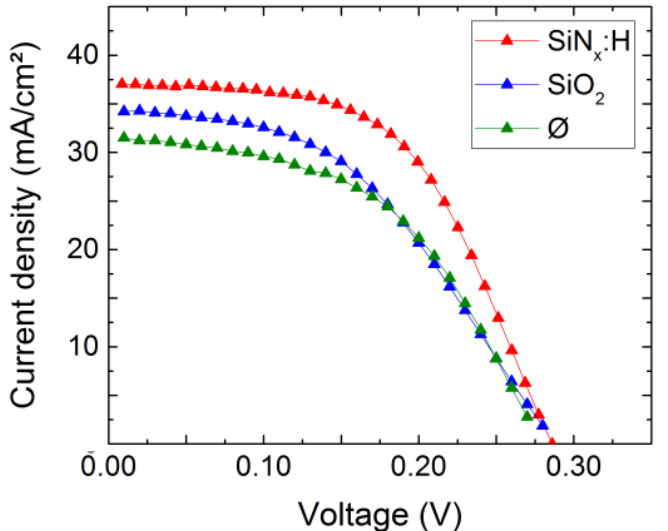

(a)

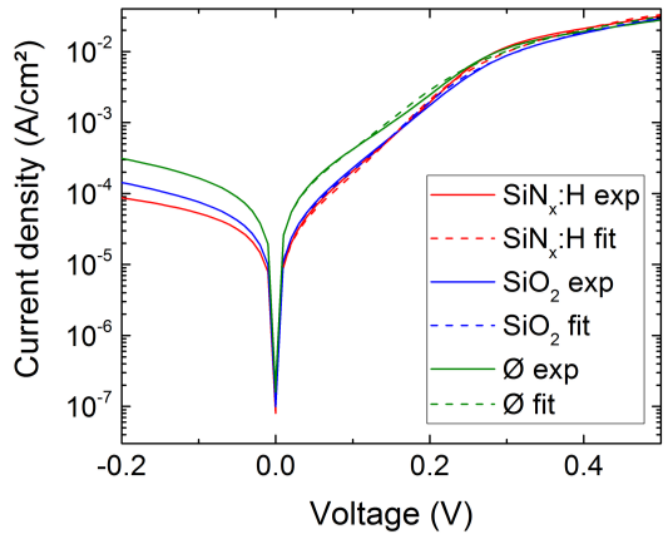

(b)

Fig.3 (a) Measured 1 sun JV curves for all types of control solar cells (5\% shading factor): with $\mathrm{SiN}_{\mathrm{x}}: \mathrm{H}_{\text {passivation (red), with } \mathrm{SiO}}$ passivation (blue) and without any passivation (green). (b) Measured (solid lines) and fitted (dashed lines) dark JV curves for all types of control solar cells (5\% shading factor): with $\mathrm{SiN}_{\mathrm{x}}: \mathrm{H}$ passivation (red), with $\mathrm{SiO}_{2}$ passivation (blue) and without any passivation (green). 


\section{GaSb-on-Si hybrid solar cell}

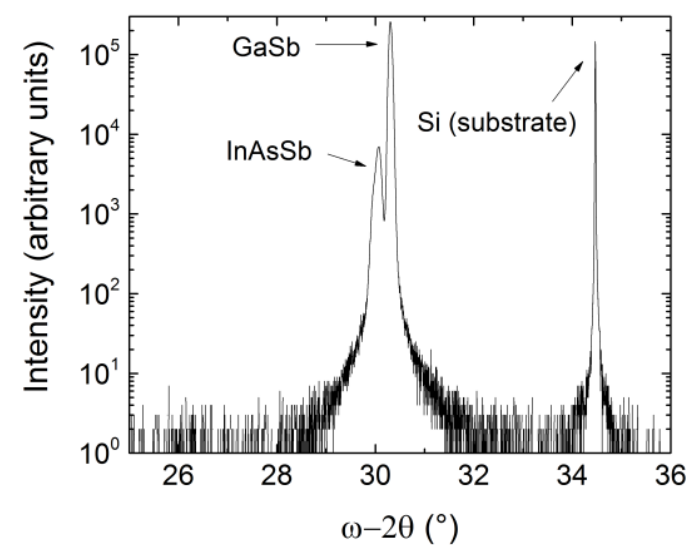

(a)

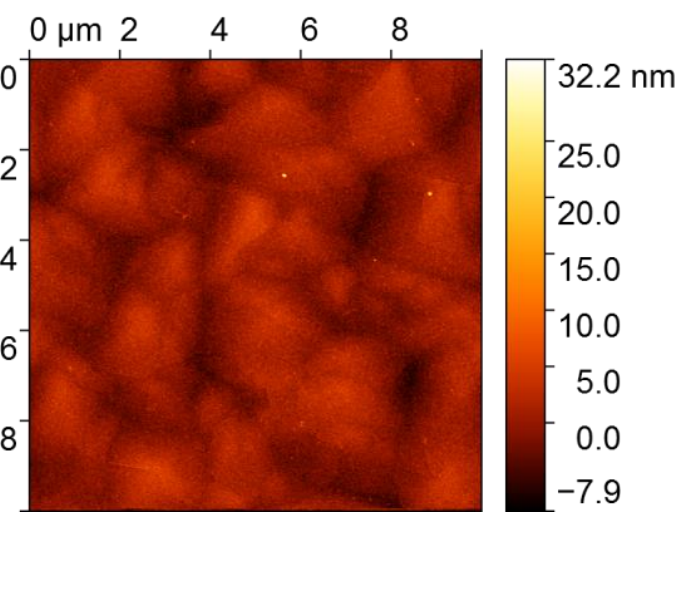

(b)

Fig.4 Material characterization of the GaSb-on-Si hybrid cell: $\omega$-2 $2 \theta$ XRD scan (a) and AFM $10 \mu \mathrm{m}$ x $10 \mu \mathrm{m}$ scan of the sample prior to processing. The estimated RMS roughness is $2 \mathrm{~nm}$.

In the case of hybrid solar cells, the epitaxial sample was studied with XRD and atomic force microscopy (AFM) prior to processing. The omega-2theta scan is shown in Fig. 4.(a) where the $\mathrm{Si}$, GaSb and InAsSb peaks can clearly be identified. The position of the GaSb peak corresponds to a $101 \%$ relaxed GaSb layer. This extra $1 \%$ is likely due to the thermal stress undergone by the material upon cooling. It can be noted that the InAsSb is not exactly lattice-matched to GaSb. A rocking curve of the sample was also measured, which allowed us to estimate the GaSb FWHM at 295 arcsec, close to the values mentioned in the literature review. The measured value is very similar to the best ones reported in [21] and around 100-200 arcsec higher than the ones reported for Ge-on-Si [22] and GaAs-on-Si [23]. Finally, the morphology of the sample was assessed with AFM (Fig. 4.(b)). The RMS roughness was evaluated at $2 \mathrm{~nm}$ (size of the scan: $10 \mu \mathrm{m} \times 10 \mu \mathrm{m}$ ), again close to the values mentioned in the literature (in the lower range of [21], still above the $<1 \mathrm{~nm}$ of [22]). As a baseline for comparison, according to our own measurements on such samples, the typical value of RMS roughness for a homoepitaxial GaSb-on-GaSb structure is in the $0.1 \mathrm{~nm}$ (sub-monolayer) range. The rougher morphology observed is expected because of the increased mismatch and because the substrate used in this work was an on axis sample. As mentioned previously, on-axis substrates generate anti-phase domains and boundaries. Anti-phase domains exhibit dissimilar growth rates, leading to rougher surfaces.

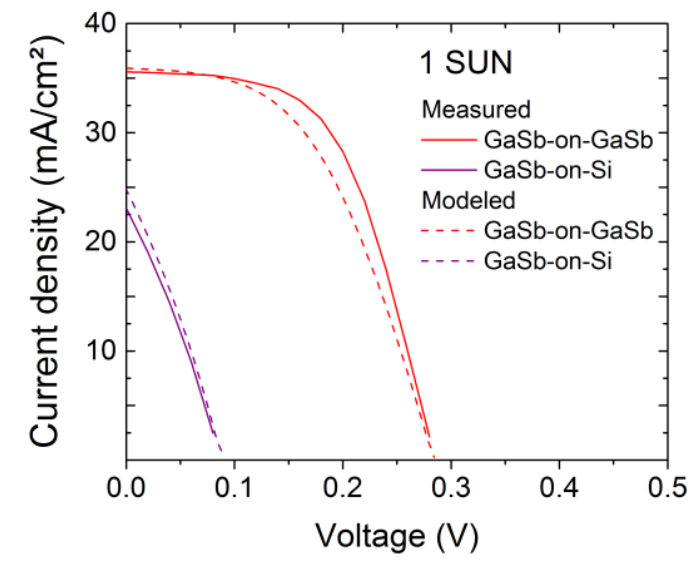

(a)

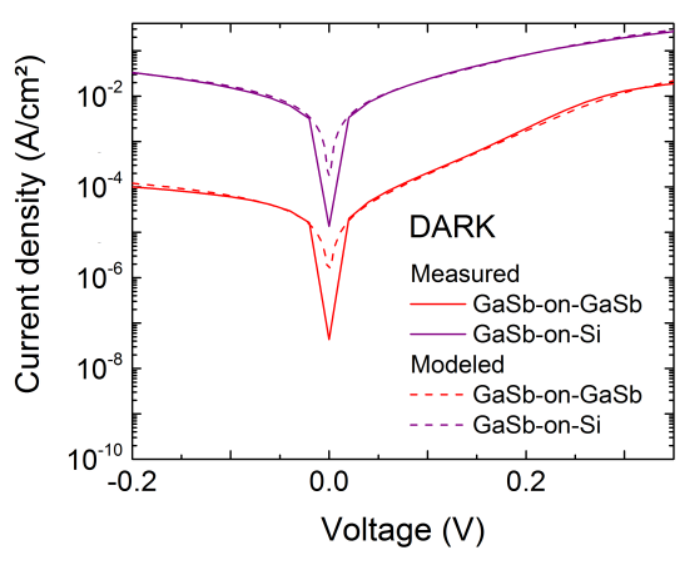

(b)

Fig.5 Measured (solid lines) and modeled (dashed lines) 1-sun (a) and dark current (b) JV curves for the GaSb-on-GaSb control solar cell (red) and GaSb-on-Si hybrid solar cell (purple).

Fig. 5 compares the JV curves of the control and the best-fabricated hybrid solar cell. The best-fabricated hybrid cell showed a significantly reduced 1-sun efficiency of $0.60 \%$ with an $80 \mathrm{mV} \mathrm{V}$ OC, a $23 \mathrm{~mA} / \mathrm{cm}^{2} \mathrm{~J}_{\mathrm{SC}}$ and a 
$31.3 \% \mathrm{FF}$. The low value of the FF and $\mathrm{V}_{\mathrm{OC}}$ reflects the high non-radiative recombination rate. Nevertheless, these parameters are close to recently reported progress on GaSb-on-GaAs cells. In particular, the measured $\mathrm{J}_{\mathrm{SC}}$ and $\mathrm{FF}$ are in between the values reported in [31] and [33]. Both references used front/back contact configurations. The front/front contact configuration used in this work circumvents the highest defect density zone, i.e., the III-Sb/Si interface. It seems that this partially compensated for the much larger lattice-mismatch $(\sim 12 \% v s \sim 8 \%$ in the case of GaSb-on-GaAs cells). The use of a thicker buffer (twice or more as thick as the ones used in [31] and [33]) also likely contributed to the lattice-mismatch compensation. However, the $\mathrm{V}_{\mathrm{OC}}$ is lower in both cases, suggesting a still higher TDD in the active zone.

TABLE III

PARAMETERS USED FOR THE CELLS SIMULATION.

\begin{tabular}{lc}
\hline \hline Parameter & Value \\
\hline Bandgap, $E_{G}$ & $0.726 \mathrm{eV}$ \\
Effective density of states in the & \\
conduction band, $N_{C, e f f}$ & $2.1 \times 10^{17} \mathrm{~cm}^{-3}$ \\
Effective density of states in the valence & \\
band, $N_{V, \text { eff }}$ & $1.8 \times 10^{19} \mathrm{~cm}^{-3}$ \\
& \\
Electron lifetime, $\tau_{n, \text { eff }}$ GaSb-on-GaSb fit & $0.4 \mathrm{~ns}$ \\
Electron lifetime, $\tau_{n, \text { eff }}$ GaSb-on-Si fit & $0.02 \mathrm{~ns}$ \\
Hole lifetime, $\tau_{p, e f f}$ GaSb-on-GaSb fit & $24 \mathrm{~ns}$ \\
Hole lifetime, $\tau_{p, e f f}$ GaSb-on-Si fit & $0.15 \mathrm{~ns}$ \\
Shunt resistance, $R_{s h}$ GaSb-on-GaSb fit & $2.0 \times 10^{3} \Omega \mathrm{cm}^{2}$ \\
Shunt resistance, $R_{s h}$ GaSb-on-Si fit & $6.7 \Omega \mathrm{cm}^{2}$ \\
Series resistance, $R_{s}$ GaSb-on-GaSb fit & $2.0 \Omega \mathrm{cm}^{2}$ \\
Series resistance, $R_{s}$ GaSb-on-Si fit & $4.0 \times 10^{-1} \Omega \mathrm{cm}^{2}$ \\
\hline \hline
\end{tabular}

In order to gain a deeper understanding, a comparison between the dark JV, 1-sun JV and quantum efficiency (QE) curves of the control and hybrid solar cells was performed on Fig. 5 and 6. To do so, we used an in-house MATLAB code solving the coupled semiconductor equations (Poisson and continuity equations) in one dimension to fit the experimental curves. The optical generation was calculated with a transfer-matrix based method, involving optical properties $(\mathrm{n}, \mathrm{k})$ taken from literature (GaSb: [34]), or from experimental ellipsometry measurements $\left(\mathrm{SiN}_{\mathrm{x}}: \mathrm{H}\right)$. The bandgap and effective density values were taken from the literature [31], [35]. The mobility values were taken from measurements made during the doping calibration stage, fitted to a simple impurity-dependent model [36]. The parameters extracted from these simulations are listed in Table III. They are valid for dark and 1-sun JV data, along with QE data. The measured and modeled JV curves are presented in Fig. 5. They are in satisfactory agreement, both for the control GaSb-on-GaSb and hybrid GaSb-on-Si solar cell. The significantly reduced values of the effective minority carrier lifetime in the hybrid solar cell reflects on the poorer material quality. The degraded value of the shunt resistance of the hybrid cell further reveals a higher number of short circuit paths. This corroborates the aforementioned material characterizations, as both the density of antiphase domains [37] and the TDD (in the case of dislocations crossing the space-charge region) [38] directly impact the value of the shunt resistance. Finally, the hybrid solar cell curve is largely dominated by shunt resistance, making the estimation of the series resistance difficult to provide. We suggest nevertheless that the improvement of the series resistance $\left(4.0 \times 10^{-1} \Omega \mathrm{cm}^{2}\right)$ compared to the control cell $\left(2.0 \Omega \mathrm{cm}^{2}\right)$ can be correlated with the benefit of using an InAsSb contact layer. Overall, the resistance values estimated with this analysis are in good agreement with previous results on thermophotovoltaic GaSb-on-GaAs cells [39], despite the increased latticemismatch.

Measured and modeled QE of both solar cells are presented on Fig. 6. The external quantum efficiency (EQE) values of the control GaSb-on-GaSb cell exceed $70 \%$ over the $1150-1540 \mathrm{~nm}$ wavelength range. The resulting maximum internal quantum efficiency (IQE) was computed to be $81.6 \%$ at $1150 \mathrm{~nm}$, indicating an efficient carrier collection in the base. Both EQE and IQE values reflect the device suitable material quality. However, the values at short wavelengths reveal important losses, pointing to poor carrier collection on the front surface. High bandgap materials such as AlGaAsSb or AlInAsSb alloys, lattice-matched to GaSb, could provide a well-suited window layer. Optimization of the emitter thickness could also improve high-energy collection, as we have used a thick 
emitter compared to standard values for photovoltaic applications ([26],[31]). The difference between the EQE and IQE curves further shows that the ARC layer thickness could be improved.

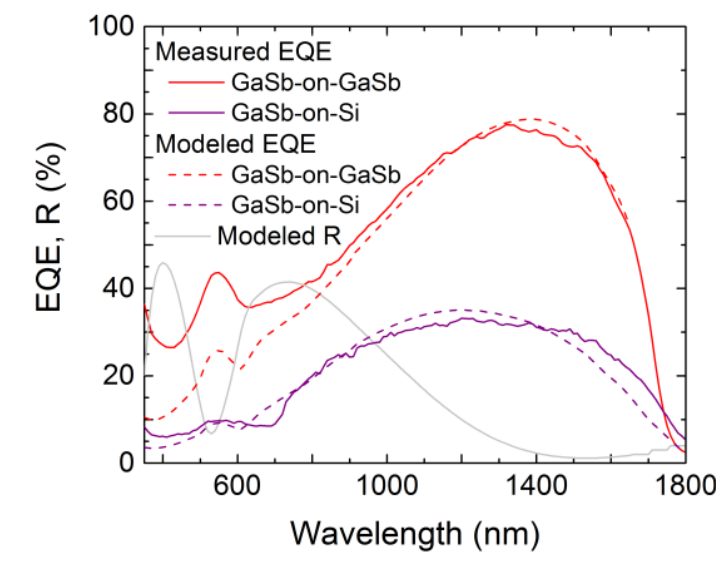

(a)

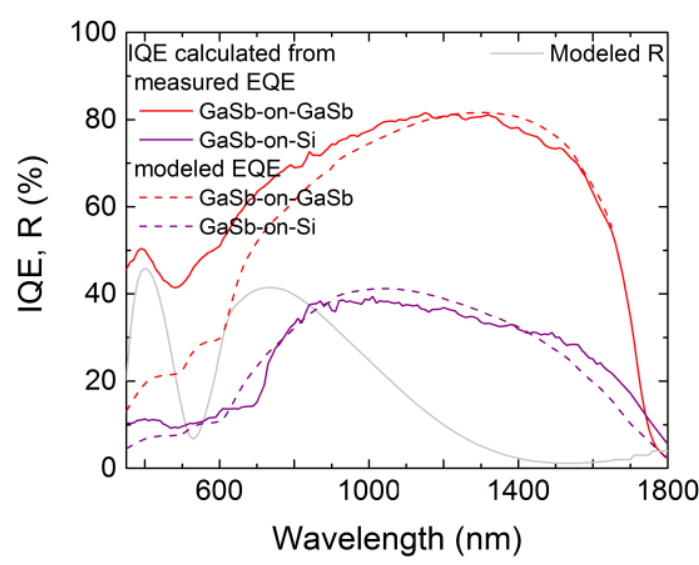

(b)

Fig.6 (a) Measured (solid lines) and modeled (dashed lines) EQE curves for the GaSb-on-GaSb control solar cell (red) and GaSb-on-Si hybrid solar cell (purple). (b) IQE curves calculated from the measured (solid lines) and modeled (dashed lines) EQE values for the GaSbon-GaSb control solar cell (red) and GaSb-on-Si hybrid solar cell (purple). The modeled reflectance is plotted in grey in both figures.

The hybrid GaSb-on-Si solar cell EQE is significantly lower (around 30\%) than the control GaSb-on-GaSb solar cell EQE. This result is consistent with a reduced effective minority carrier lifetime in the emitter and base. The one-dimensional simulation does not allow dissociating bulk from surface recombination, as it does not take into account surface recombination occurring at the mesa sidewalls. However, since the structure (thicknesses, doping) and passivation of the two cells are similar, we can consider their surface recombination velocities to be equivalent. The much poorer effective carrier lifetimes estimated in the hybrid cell (one or two orders of magnitude lower) can consequently be attributed to a higher volumetric non-radiative recombination rate. This would imply that the degradation of the hybrid cell performance dominantly stems from losses in the bulk, likely induced by the elevated TDD. Using a simple model by Yamaguchi et al, we can approximate the minimum TDD at the bottom of the hybrid solar cell by $4 /\left[\pi^{3} \mathrm{~L}_{p}{ }^{2}\right]$, where $\mathrm{L}_{\mathrm{p}}$ is the minority carrier diffusion length [40]. We compute a minimum TDD in the $2 \times 10^{8} \mathrm{~cm}^{-2}$ range, higher than in the case of [31]. This value is still far from the target. Other works, mentioned in the literature review, have employed much thicker buffer layers $(10-15 \mu \mathrm{m} v s 1 \mu \mathrm{m}$ used in this work) to achieve TDDs in the $10^{6} \mathrm{~cm}^{-2}$ range. One avenue of research would then be to increase the $\mathrm{GaSb}$ buffer thickness below the InAsSb contact layer to move the active region further away from the high defect density region. We do not consider at this point the SLS and TCA techniques, for the sake of simplicity. Finally, during the hybrid solar cells processing, it appeared that significant oxidation occurred at the surface of the samples. Such oxidation impedes efficient surface passivation, facilitates surface recombination and hinders smooth deposition of the metallic contacts. Future work will focus on more thorough deoxidation processes or insitu thermal treatments before the passivation and contact layers deposition.

\section{Conclusion:}

We reported here on the first growth and processing of a hybrid GaSb-on-Si solar cell. In this perspective we have first performed a study of stand-alone GaSb control cells, achieving a satisfying 1-sun efficiency of 5.90\%. We have then adapted the fabrication procedure to hybrid GaSb-on-Si solar cells to circumvent the high defect density region at the hetero-interface. Although the electrical performance of the hybrid cell is - as expected - drastically reduced compared to the control cell, the obtained results are close to the ones obtained for GaSb-on-GaAs solar cells despite a much larger lattice-mismatch. We have further discussed possible improvements to both the growth and process flow. This work constitutes the first building blocks toward the development of III-Sb-based solar cells onto Si. A good quality GaSb solar cell also paves the way to the development of all lattice-matched multijunction solar cell on GaSb. 


\section{Acknowledgments}

This work was partially supported by the French "Investment for the Future" program (EquipEx EXTRA, ANR11-EQPX-0016, ANR-10-LABX-22-01-SOLSTICE), the European Union's Horizon 2020 research program under the Marie Sklodowska-Curie grant agreement No 641899.

\section{References}

[1] M. A. Green, Y. Hishikawa, E. D. Dunlop, D. H. Levi, J. Hohl-Ebinger and A W.Y. Ho-Baillie, "Solar cell efficiency tables (version 52)," Progress in Photovoltaics: Research and Applications, vol. 26, no. 7, pp 3-12, June 2018.

[2] National Renewable Energy Laboratory. (2018, Apr.). "Best research-cell efficiencies". Available: http://www.nrel.gov/pv/assets/images/efficiency-chart.png

[3] A. Slade, V. Garboushian, "27.6\% efficient silicon concentrator solar cells for mass production," presented at the 15 th International Photovoltaic Science and Engineering Conference, Beijing. 2005.

[4] Soitec. (Financial press release of May 21 $1^{\text {st }}, 2015$ ). "Soitec divests its solar system business to refocus on its core semiconductor activities.” Available: http://www.soitec.com/media/financialreleasedocuments/86/file/soitec_concensolar_pr_21may2015_final_en.pdf

[5] K. A. Horowitz, M. Woodhouse, H. Lee and G. P. Smestad, "A bottom-up cost analysis of a high concentration PV module," AIP Conference Proceedings, vol. 1679, no. 1, pp. 10001-1-6, Sep. 2015.

[6] M. Yamaguchi, K. H. Lee, K. Araki, N. Kojima and Y. Ohshita, "Potential and activities of III-V/Si tandem solar cells," ECS Journal of Solid State Science and Technology, vol. 5, no. 2, pp. Q68-Q73, Jan. 2016.

[7] K. N. Yaung,, M. Vaisman, J. Lang. and M.L. Lee, "GaAsP solar cells on GaP/Si with low threading dislocation density," Applied Physics Letters, vol. 109, no. 3, pp. 032107-1-5, Jul. 2016.

[8] M. Vaisman, S. Fan, K. N. Yaung, E. Perl, D. Martín-Martín, Z. J. Yu, M. Leilaeioun, Z. C. Holman and M. L. Lee, “15.3\% Efficient GaAsP solar cells on GaP/Si templates," ACS Energy Letters, vol. 2, no. 8, pp. 1911-1918, Aug. 2017.

[9] S.M. Vernon, S. P. Tobin, V. E. Haven, C. Bajgar, T. M. Dixon, M. M. Al-Jassim, R.K. Ahrenkiel and K.A. Emery, "Efficiency improvements in GaAs-on-Si solar cells," in Proc. of 20th IEEE Photovoltaic Specialists Conference, pp. 481-485, 1988.

[10] S.M. Vernon, S. P. Tobin, V. E. Haven, L. M. Geoffroy and M. M. Sanfacon, "High-efficiency concentrator cells from GaAs on Si," in Conference record of the 22nd IEEE, pp. 353-357, Oct. 1991.

[11] Y. Ohmachi, Y. Kadota, Y. Watanabe and H. Okamoto, "High quality GaAs on Si and its application to a solar cell," MRS Online Proceedings Library Archive, vol. 144, pp. 297-302, 1988.

[12] C. L. Andre, J. A. Carlin, J. J. Boeck1, D. M. Wilt, M. A. Smith, A. J. Pitera, M. L. Lee, E. A. Fitzgerald and S. A. Ringel, "Investigations of high-performance GaAs solar cells grown on $\mathrm{Ge}_{-} \mathrm{Si}_{1-\mathrm{x}} \mathrm{Ge}_{\mathrm{x}}-\mathrm{Si}$ substrates," Journal of applied physics, vol. 58, no. 1, pp. 1055-1060, Jun. 2005.

[13] C. L. Andre, D. M. Wilt , A. J. Pitera, M. L. Lee, E. A. Fitzgerald and S. A. Ringel, "Impact of dislocation densities on n+/p and p $+/ n$ junction GaAs diodes and solar cells on SiGe virtual substrates," IEEE Transactions on Electron Devices, vol. 52, no. 6, pp. 014502-15, Jun. 2005.

[14] Y. Wang, Z. Ren, M. Thway, K. Lee, S. F. Yoon, I. M. Peters, T. Buonassisi, E. A. Fizgerald, C.S Tan and K. H. Lee, "Fabrication and characterization of single junction GaAs solar cells on Si with As-doped Ge buffer," Solar Energy Materials and Solar Cells, vol. 172, pp. 140-144, Dec. 2017.

[15] A. Jallipalli, M. N. Kutty, G. Balakrishnan, J. Tatebayashi, N. Nuntawong, S. H. Huang, L. R. Dawson, D. L. Huffaker, Z. Mi, and P. Bhattacharya, "1.54 $\mu \mathrm{m} \mathrm{GaSb} / \mathrm{AlGaSb}$ multi-quantum-well monolithic laser at $77 \mathrm{~K}$ grown on miscut Si substrate using interfacial misfit arrays," Electronics Letters, vol. 43, no. 22, pp. 1198-1199, Oct. 2007.

[16] K. M. Ko, J. H. Seo, D. E. Kim, S. T. Lee, Y. K. Noh, M. D. Kim, and J. E. Oh, "The growth of a low defect InAs HEMT structure on Si by using an AlGaSb buffer layer containing InSb quantum dots for dislocation termination," Nanotechnology, vol. 20, no. 22, pp. 225201-1-8, May 2009.

[17] A. Castellano, L. Cerutti, J. B. Rodriguez, G. Narcy, A. Garreau, F. Lelarge, and E. Tournié, "Room-temperature continuous-wave operation in the telecom wavelength range of GaSb-based lasers monolithically grown on Si," APL Photonics, vol. 2, no. 6, pp. 061301-1-5, Jun. 2017.

[18] H. Nguyen-Van, A. N. Baranov, Z. Loghmari, L. Cerutti, J. B. Rodriguez, J. Tournet, G. Narcy, G. Boissier, G. Patriarche, M. Bahriz and E. Tournié, "Quantum cascade lasers grown on silicon," Scientific reports, vol. 8, pp. 7206-1-8, May 2018.

[19] S. H. Huang, G. Balakrishnan, A. Khoshakhlagh, L. R. Dawson, and D. L. Huffaker, "Simultaneous interfacial misfit array formation and antiphase domain suppression on miscut silicon substrate," Applied Physics Letters, vol. 93, no. 7, pp. 071102-1-3, Aug. 2008.

[20] K. Akahane, Kouichi, N. Yamamoto, S. I. Gozu, A. Ueta, and N. Ohtani, "Initial growth stage of GaSb on Si (0 0 1) substrates with AlSb initiation layers," Journal of crystal growth, vol. 283, pp. 297-302, Oct. 2005.

[21] J. B. Rodriguez, K. Madiomanana, L. Cerutti, A. Castellano, and E. Tournié, "X-ray diffraction study of GaSb grown by molecular beam epitaxy on silicon substrates," Journal of crystal growth, vol. 439, pp. 33-39, Apr. 2016.

[22] K. W. Shin, H. W. Kim, J. Kim, C. Yang, S. Lee, and E. Yoon, "The effects of low temperature buffer layer on the growth of pure Ge on Si (001)," Thin Solid Films, vol. 518, no. 22, pp. 6496-6499, Sep. 2010.

[23] Y. B. Bolkhovityanov and O. P. Pchelyakov, "GaAs epitaxy on Si substrates: modern status of research and engineering," PhysicsUspekhi, vol. 51, no. 5, pp. 437-456, 2008.

[24] J. B. Rodriguez, L. Cerutti, G. Patriarche, L. Largeau, K. Madiomanana, and E. Tournié, "Characterization of antimonide based material grown by molecular epitaxy on vicinal silicon substrates via a low temperature AlSb nucleation layer," Journal of Crystal Growth, vol. 477, pp. 65-71, Nov. 2017.

[25] J. Tournet, Y. Rouillard and E. Tournié, “AlInAsSb for GaSb-based multi-junction solar cells," in Proc. of SPIE Photonics West Opto Conference, Physics, Simulation and Photonic Engineering of Photovoltaic Devices VII, 105270A-1-9, Feb. 2018.

[26] M. P. Lumb, S. Mack, K. J. Schmieder, M. González, M. F. Bennett, D. Scheiman, M. Meitl, B. Fisher, S. Burroughs, K. T. Lee and J. A. Rogers, "GaSb-based solar cells for full solar spectrum energy harvesting," Advanced Energy Materials, vol. 7, no. 20, pp. 17003451-9, Oct. 2017. 
[27] S. Parola, A. Vauthelin, F. Martinez, J. Tournet, J. El Husseini, J. Kret, E. Quesnel, Y. Rouillard, E. Tournié and Y. Cuminal, "Investigation of antimonide-based semiconductors for high-efficiency multi-junction solar cells," in Proc. of the 2018 WCPEC-7 (PVSC45), to be published.

[28] O. Dier, C. Lauer, and M-C. Amann, "n-InAsSb/p-GaSb tunnel junctions with extremely low resistivity,” Electronics Letters, vol. 42, no. 7, pp. 419-420, Mar. 2006.

[29] A. S. Bracker, M. J. Yang, B. R. Bennett, J. C. Culbertson, and W. J. Moore, "Surface reconstruction phase diagrams for InAs, AlSb, and GaSb," Journal of Crystal Growth, vol. 220, no. 4, pp.384-392, Dec. 2000.

[30] K. Madiomanana, M. Bahri, J. B. Rodriguez, L. Largeau, L. Cerutti, O. Mauguin, A. Castellano, G. Patriarche, and E. Tournié, "Silicon surface preparation for III-V molecular beam epitaxy," Journal of Crystal Growth, vol. 413, pp. 17-24, Mar. 2015.

[31] G. T. Nelson, B. C. Juang, M. A. Slocum, Z. S. Bittner, R. B. Laghumavarapu, D. L. Huffaker, and S. M. Hubbard, "GaSb solar cells grown on GaAs via interfacial misfit arrays for use in the III-Sb multi-junction cell," Applied Physics Letters, vol. 111, no. 23, pp. 231104-1-5, Dec. 2017.

[32] E.Q. Macabebe and E. E. Van Dyk, "Parameter extraction from dark current-voltage characteristics of solar cells," in South African Journal of Science, vol. 104, no. 9-10, pp.401-404, Oct. 2008

[33] A. Mansoori, S. J. Addamane, E. J. Renteria, D. M. Shima, M. Behzadirad, E. Vadiee, C. Honsberg, and G. Balakrishnan. "Reducing threading dislocation density in GaSb photovoltaic devices on GaAs by using AlSb dislocation filtering layers," Solar Energy Materials and Solar Cells, vol. 185, pp. 21-27, Oct. 2018.

[34] E. D. Palik, "Handbook of Optical Constants of Solids," Academic Press, 1985.

[35] http://www.ioffe.ru/SVA/NSM/Semicond/GaSb/bandstr.html, consulted in June 2018.

[36] L. Tang, L. M. Fraas, Z. Liu, C. Xu, and X. Chen. "Performance improvement of the GaSb thermophotovoltaic cells with n-type emitters," IEEE Transactions on Electron Devices, vol. 62, no. 9, pp. 2809-2815, Sept. 2015.

[37] B. Galiana, I. Rey-Stolle, I. Beinik, C. Algora, C. Teichert, J. M. Molina-Aldareguia and P. Tejedor, "Characterization of antiphase domains on GaAs grown on Ge substrates by conductive atomic force microscopy for photovoltaic applications," Solar Energy Materials and Solar Cells, vol. 95, no. 7, pp. 1949-1954, Jul. 2011.

[38] H. El Ghitani, M. Pasquinelli, and S. Martinuzzi. "Influence of dislocations on photovoltaic properties of multicrystalline silicon solar cells," Journal de Physique III, vol. 3, no. 10, pp.1941-1946, Oct. 1993.

[39] B. C. Juang, R. B. Laghumavarapu, B. J. Foggo, P. J. Simmonds, A. Lin, B. Liang and D. L. Huffaker, "GaSb thermophotovoltaic cells grown on GaAs by molecular beam epitaxy using interfacial misfit arrays," Applied Physics Letters, vol. 106, no. 11, pp. 111101-1-5, Mar. 2015.

[40] M. Yamaguchi and C. Amano, "Efficiency calculations of thin-film GaAs solar cells on Si substrates," Journal of Applied Physics, vol. 58, no. 9, pp.3601-3606, Nov. 1985. 